\title{
The effect of acute or long-term genistein administration on the ischemia/reperfusion-induced arrhythmia in rats
}

\begin{abstract}
The cardiovascular disease and sudden death due to the occlusion of coronary artery increases in women after menopause. In this study, it was aimed to research the effect of genistein that has been used to decrease the symptom of menopause in women, on the ischemia reperfusion induced arrhythmias. 64 Female and 12 male, 6-7months old Sprague Dowley rats were used in this study. Genistein was applied daily for four weeks in $100 \mathrm{ug} / \mathrm{kg}$ dose intraperitoneally in one group and acutely in other group, before reperfusion in $1 \mathrm{mg} / \mathrm{kg}$ dose intravenously. Six minutes myocardial ischemia were produced by the ligation of ramus interventricularis branch of left coronary artery (LAD) and six minutes of reperfusion by releasing of this artery. The type and duration of arrhythmias and the blood pressure recorded in every group during ischemia and reperfusion were analyzed and compared by using one-way ANOVA test and the incidence of arrhythmia and the survival rate by Ki square test. Genistein had no effect on the ischemia and reperfusion induced arrhythmias in male and ovariectomized female rats.
\end{abstract}

Keywords: ischemia, reperfusion, myocardial, arrhythmia, genistein
Volume 5 Issue 6 - 2018

\author{
Omer Bozdogan,' Didem Eksioglu,' Firdevs \\ Erim,' Salih Tunc Kaya, ${ }^{2}$ Ogulcan Talat \\ Ozaslan,' Selcuk Yasar' \\ 'Department of Biology, Faculty of Arts and Science, Abant Izzet \\ Baysal University, Turkey \\ ${ }^{2}$ Department of Biology, Faculty of Arts and Science, Duzce \\ University, Turkey
}

\begin{abstract}
Correspondence: Omer Bozdogan, Biology Department, Faculty of Arts and Science, Abant Izzet Baysal University, Bolu, Turkey, Fax 0903742534506, Tel 0903742541000,
\end{abstract}

Email bozdagan_o@ibu.edu.tr

Received: November 13, 2017 | Published: November 21, 2018

\section{Introduction}

Epidemiologic studies show that appearance of coronary artery disease in women rises after postmenopausal period. ${ }^{1,2}$ This situation is attributed to decreasing estrogen level in postmenopausal period. ${ }^{3}$ Phytoestrogen have been used to replace the estrogen in the post menopause period in women. ${ }^{4}$ Genistein is the most known phytoestrogen. It binds to estrogen receptor and act as like estrogen. ${ }^{5}$ In various studies, the cardio protective effect of genistein was shown. ${ }^{6,7}$ Phytoestrogen including genistein and others decreases symptom appearing in postmenopausal period; decreases the risk of heart attack, blood cholesterol, thrombocyte aggregation and increases angiogenesis and vasodilatation. ${ }^{8}$ It was indicated that genistein shows estrogenic effect in lower doses and antiestrogenic effect in higher doses. ${ }^{5}$ Genistein in lower doses decreases the ventricular arrhythmia and infarct sizes in ovariectomized female rats. ${ }^{1}$ Otherwise, it was also shown that genistein abolishes the antiarrhythmic effect of ischemic preconditioning in Langendorf perfused rats. ${ }^{9}$ Söylemez et al. ${ }^{10}$ observed that genistein alone was not found to be effective on the ischemia-induced arrhythmia. Long-term administration of genistein and its effects on the ischemia or reperfusion induced arrhythmia were not studied previously. However, in a study by Mendelsohn \& Karas. ${ }^{2}$ It is reported that administration of genistein in lower doses for two days increased the contractility of myocardium and decreased contractility in higher doses $(150 \mathrm{uM})$. Moreover, there is only one study related with the acute effect of genistein on the reperfusioninduced arrhythmia. ${ }^{1}$ That is why it was aimed to research the effect of both long term and acute genistein administration on the ischemia and reperfusion induced arrhythmia in ovariectomized female and male rats in this study.

\section{Materials and methods}

\section{Animals}

In this study, 64 female, 12 male animals of 6-7 months old were used. The effect of genistein was researched in two group of animals. First group consisting 39 rats, genistein was given just before myocardial reperfusion after six minutes of coronary ligation (Figure 1). In the Second group consisting of 37 rats, genistein was given every morning for 4 weeks. Acute effect of genistein was also researched in male animals (Figure 2). The effect of genistein in female animals was researched in ovariectomized, sham operated and unovariectomized animals. The animals were fed with commercial rat pellet food and they drank tap water ad libitum. The animals were kept in light for 12 hours and in dark for another 12 hours. The animals having arrhythmias or having mean arterial pressure below $60 \mathrm{mmHg}$ before coronary ligation was discarded from the experiment. Recording and animal ventilation were stopped at the end of reperfusion. Animals with no respiration after removing the ventilator were accepted as dead and discarded from evaluation. All the animals were handled according to the protocol approved by the ethical committee for the protection of animal research of the Abant Izzet Baysal University, Bolu, Turkey, protocol numbers; 2012/49, 2014/30.

\section{Surgical operation}

Ovariectomy and sham operation were made under the ketamine $(90 \mathrm{mg} / \mathrm{kg})$ and xylazine $(10 \mathrm{mg} / \mathrm{kg})$ anesthesia. Following the operation, animals were waited for two weeks for their complete recovery and then coronary ligation and reperfusion were made. Ovariectomy were made by bilateral abdominal incision and by the ablation of ovary. In the sham operated animals, only abdominal 
incisions were made but ovary remained intact with the animals. Animals were anesthetized with urethane $(1.2 \mathrm{~g} / \mathrm{kg})$ for the operation of coronary artery ligation and reperfusion. Descending branch of left coronary artery (LAD) was ligated by tightening the silk thread by forming a bowknot to produce myocardial ischemia for 6 minutes and reperfusion was made by loosening the thread for 6 minutes. ${ }^{11}$ At the end of the coronary ligation and reperfusion, the heart was perfused by the way of the aorta by giving firstly sodium chloride $(\mathrm{NaCI})$ and then ethanol to determine the risk of infarct zone. Then the non-perfused area that seen red in color was separated from the perfused area that is seen white in color. The non-perfused area and perfused area were weighted alone and together. The percentage of non-perfused area in respect to total weight of ventricle was calculated. This calculation was called as the risk of infarct zone.

\section{Drug administration}

Genistein was dissolved in DMSO and given intraperitoneally daily in $100 \mathrm{ug} / \mathrm{kg} / 0.1 \mathrm{ml}$ dose during four weeks and in the second group intravenously $1 \mathrm{mg} / \mathrm{kg} / 0.1 \mathrm{ml}$ from tail vein just before reperfusion.

\section{Recording}

ECG recording was measured by subcutaneous needle electrodes. ECG and blood pressure were recorded during ischemia and reperfusion by computerized recording system (Biopac System Inc Goleta CA USA, Turkish representative Commat-Ankara/Turkey) using BSL Pro 3.7 software. The type of ventricular arrhythmia, the duration of each type of arrhythmia and total arrhythmia were determined in six minutes ischemia and six minutes reperfusion period. Arrhythmias were classified according to the Lambeth conventions. ${ }^{12}$ According to the type and duration of arrhythmia, an arrhythmia score was determined..$^{13}$ The heart rate and blood pressure before and following ligation and reperfusion at $1 \mathrm{st}, 3 \mathrm{rd}$ and 5 th minutes were determined from ECG and blood pressure recordings. Time scale and recording in acute and long-term genistein administered group were shown in Figure $1 \& 2$.

\section{Biochemical analysis}

Plasma estradiol concentration level was measured in all female rats using commercial ELISA kits according to the instruction given by the manufacturer (Sunred bio, Shanghai-China).

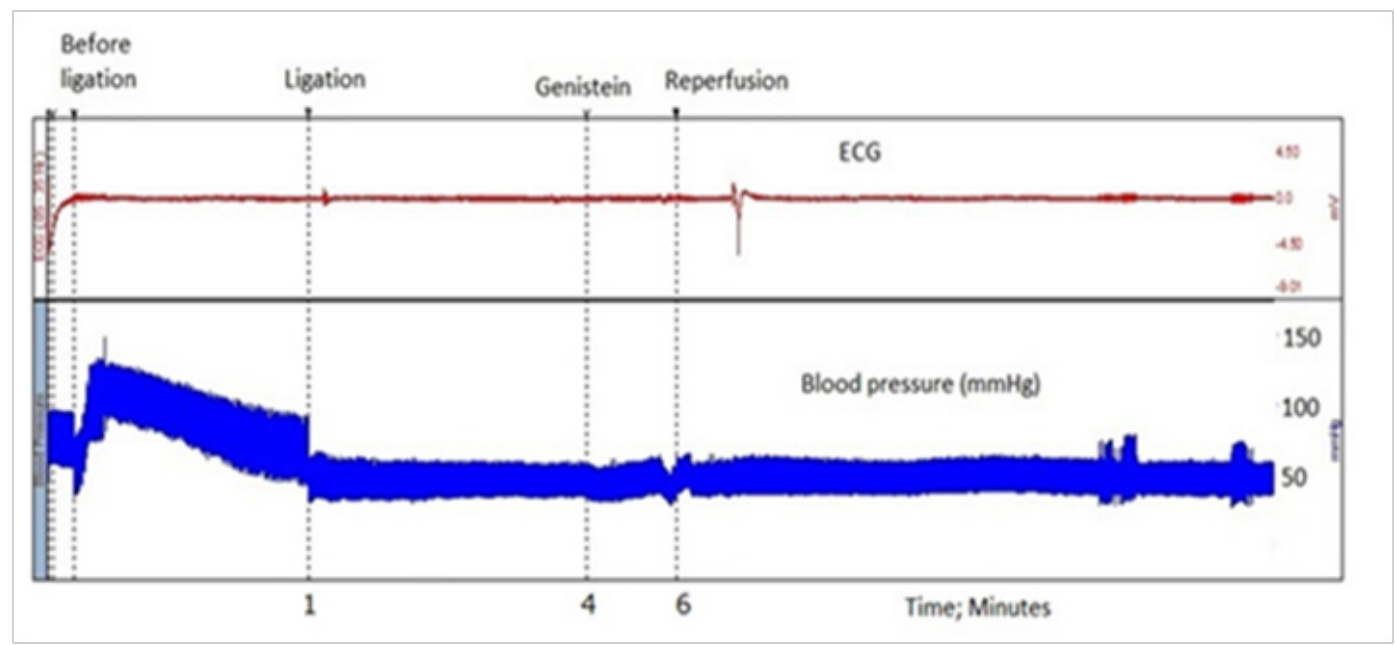

Figure I Time scale of ischemia, reperfusion and genistein administration in group I.

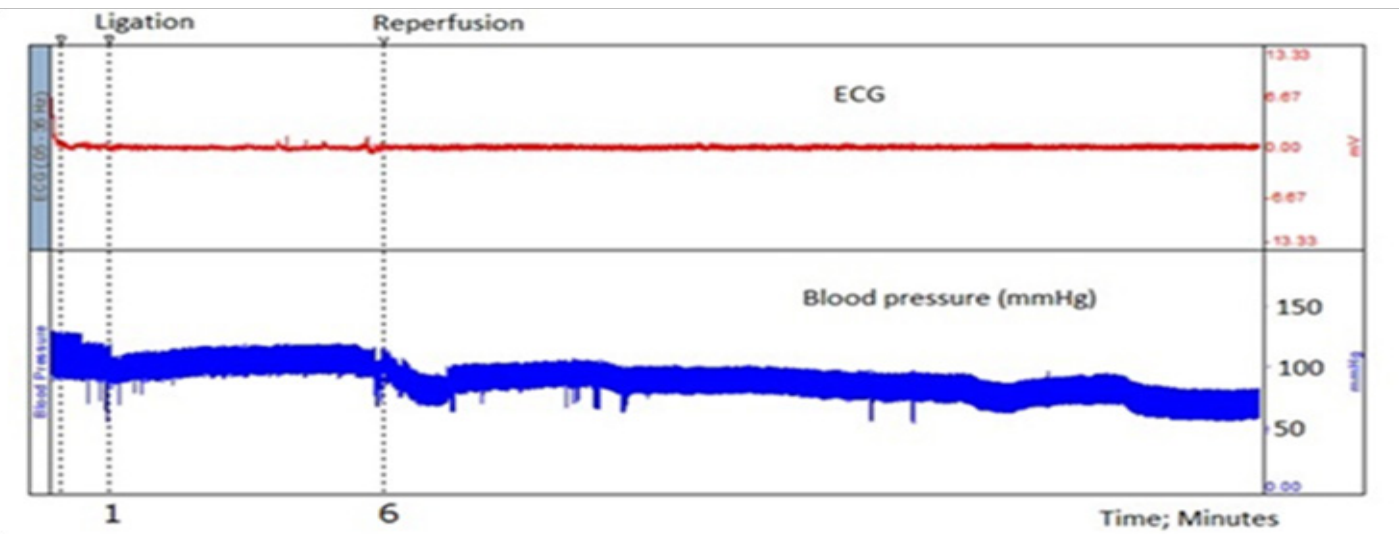

Figure 2 Time scale of ischemia and reperfusion in group 2. 


\section{Statistical analysis}

The mean and standard errors were calculated for all parameters including heart rate and blood pressure. The duration of arrhythmias and arrhythmia scores were compared by the analysis of variance with one-way ANOVA combined with the LSD post hoc test. Furthermore, the survival rate and the incidence of arrhythmias were compared by chi-square test (Fisher exact test, two-tailed).

\section{Results}

ST segment elevation or QRS changes were observed following coronary artery ligation in all groups. Risk of infarct zone was not different among groups. Basal blood pressure (before ligation) was higher only in sham+genistein group than the sham and control groups $(\mathrm{p}<0.05)$ in animals introduced with long term genistein (Table 1). Blood pressure and heart rate during ischemia and reperfusion did not change significantly in respect to control groups. Similarly, acute genistein administration did not exhibit any effect that changes the blood pressure and heart rate during reperfusion period in female and male animals in respect to their controls. The estradiol level decreased in ovariectomized rats (Table 2). Arrhythmic period, total arrhythmia, the length of each type of arrhythmia, the arrhythmia score and the incidence of arrhythmia determined from recorded ECG during ischemia (Table 3) and reperfusion period (Table 4) were not different in long term genistein administered rats. In acutely genistein administered group, the other type of arrhythmia occurred during reperfusion decreased in overiectomized female and sham operated female in respect to female control, but these arrhythmia was also lesser in overiectomized but not genistein administered group (Table 5). Genistein was not effective on the reperfusion-induced arrhythmia in males. The score of arrhythmia and the incidence of arrhythmia in overiectomized female and also male in respect to their control did not change significantly with the acute administration of genistein.

Table I The blood pressure and heart rate during six minutes of ischemia and six minutes reperfusion

\begin{tabular}{|c|c|c|c|c|c|c|c|c|c|c|c|}
\hline \multirow[t]{2}{*}{ Groups } & \multirow[b]{2}{*}{$\mathbf{N}$} & \multicolumn{5}{|c|}{ Heart rate (Beat / min) } & \multicolumn{5}{|c|}{ Blood pressure (mmHg) } \\
\hline & & Basal & Lig & 1 & 3 & 5 & Basal & Lig & 1 & 3 & 5 \\
\hline Control $_{(\mathrm{a})}$ & 8 & $345 \pm 23$ & $330 \pm 29$ & $342 \pm 26$ & $339 \pm 28$ & $334 \pm 23$ & $101 \pm 5$ & $66 \pm 3$ & $68 \pm 5$ & $68 \pm 6$ & $66 \pm 6$ \\
\hline $\begin{array}{l}\text { Control + } \\
\text { Gen }\end{array}$ & 7 & $328 \pm 21$ & $345 \pm 28$ & $335 \pm 19$ & $374 \pm 30$ & $352 \pm 26$ & $107 \pm 8$ & $67 \pm 7$ & $83 \pm 5$ & $70 \pm 4$ & $64 \pm 5$ \\
\hline $\mathrm{Ovx}_{(\mathrm{c})}^{(\mathrm{b})}$ & 7 & $287 \pm 23$ & $327 \pm 26$ & $319 \pm 28$ & $321 \pm 30$ & $328 \pm 32$ & $123 \pm 15$ & $81 \pm 11$ & $82 \pm 11$ & $76 \pm 8$ & $77 \pm 10$ \\
\hline $\mathrm{Ovx}+\mathrm{Gen}_{\text {(d) }}$ & 7 & $326 \pm 23$ & $338 \pm 40$ & $350 \pm 30$ & $362 \pm 17$ & $335 \pm 42$ & $112 \pm 4$ & $66 \pm 6$ & $77 \pm 4$ & $67 \pm 7$ & $65 \pm 8$ \\
\hline $\operatorname{Sham}_{(\mathrm{e})}$ & 5 & $297 \pm 12$ & $345 \pm 22$ & $323 \pm 41$ & $347 \pm 29$ & $341 \pm 30$ & $103 \pm 9$ & $63 \pm 5$ & $63 \pm 3$ & $67 \pm 6$ & $67 \pm 7$ \\
\hline $\operatorname{Sham}+\mathrm{Gen}_{(\mathrm{f})}$ & 5 & $337 \pm 21$ & $387 \pm 20$ & $373 \pm 28$ & $385 \pm 24$ & $356 \pm 16$ & $132 \pm 5^{\text {ae }}$ & $84 \pm 3$ & $82 \pm 5$ & $81 \pm 7$ & $74 \pm 12$ \\
\hline
\end{tabular}

Abbreviations: Basal, I min before ligation; Lig, at the time point of ischemia; 0 I, I minute after ligation; 03, 3 minutes after ligation; 05, 5 minutes after ligation Values were represented as mean \pm standard error. ap $<0.05$; different from control, ep $<0.05$; different from sham

Table 2 Plasma estradiol levels in female rats $(\mathrm{ng} / \mathrm{ml})$

\begin{tabular}{lll}
\hline Groups & N & Plasma estradiol $(\mathbf{n g} / \mathbf{m l})$ \\
\hline Control & 6 & $109 \pm 3$ \\
Control+Gen & 6 & $95 \pm 4$ \\
Ovx & 6 & $68 \pm 10^{*}$ \\
Ovx + Gen & 6 & $95 \pm 4$ \\
Sham & 6 & $99 \pm 4$ \\
Sham + Gen & 6 & $97 \pm 4$ \\
\hline
\end{tabular}

${ }^{*} \mathrm{p}<0.05$; different from control

Table 3 The type and duration of arrhythmia during six minutes of coronary ligation in long-term genistein administered group in female rats

\begin{tabular}{lllllllll}
\hline \multirow{2}{*}{ Groups } & N & Arrhythmic period (min) & \multicolumn{2}{c}{ Duration of arrhythmia (min) } & & \multicolumn{2}{c}{$\begin{array}{c}\text { Score of } \\
\text { arrhythmia }\end{array}$} \\
\cline { 4 - 7 } & & & VF & VT & Others & Total & Bradycardia \\
\hline Control & 8 & $0.3 \pm 0.3$ & $0 \pm 0$ & $0 \pm 0$ & $0.3 \pm 0.1$ & $0.2 \pm 0.1$ & $0 \pm 0$ & $0.3 \pm 0.2$ \\
Control+Gen & 7 & $0 \pm 0$ & $0 \pm 0$ & $0 \pm 0$ & $0.1 \pm 0.1$ & $0.1 \pm 0.1$ & $0 \pm 0$ & $0.4 \pm 0.2$ \\
Ovx & 7 & $0.1 \pm 0.1$ & $0 \pm 0$ & $0 \pm 0$ & $0.7 \pm 0.5$ & $0.7 \pm 0.5$ & $0 \pm 0$ & $0.4 \pm 0.2$ \\
Ovx+Gen & 7 & $0.8 \pm 0.8$ & $0.7 \pm 0.7$ & $5.3 \pm 5.3$ & $1.8 \pm 1.5$ & $7.9 \pm 7.6$ & $0 \pm 0$ & $0.9 \pm 0.4$ \\
Sham & 5 & $0.2 \pm 0.1$ & $0 \pm 0$ & $0 \pm 0$ & $2.2 \pm 1.8$ & $2.2 \pm 1.8$ & $0 \pm 0$ & $0.6 \pm 0.2$ \\
Sham+Gen & 5 & $0.9 \pm 0.8$ & $0 \pm 0$ & $5.2 \pm 5.2$ & $2.1 \pm 1.9$ & $7.3 \pm 7.1$ & $0 \pm 0$ & $0.8 \pm 0.4$ \\
\hline
\end{tabular}

Abbreviations: N, number of animals; $\mathrm{VF}$, ventricular fibrillation; $\mathrm{VT}$, ventricular tachycardia; Others; ventricular premature contraction, bigeminy, salvo. Values were represented as mean \pm standard error 
Table 4 The type and duration of arrhythmias during 6 minutes of reperfusion in long-term genistein administered group in female rats

\begin{tabular}{|c|c|c|c|c|c|c|c|c|}
\hline \multirow[t]{2}{*}{ Groups } & \multirow[t]{2}{*}{$\mathbf{N}$} & \multirow[t]{2}{*}{$\begin{array}{l}\text { Arrhythmic period } \\
\text { (minute) }\end{array}$} & \multicolumn{5}{|c|}{ Duration of arrhythmia (second) } & \multirow[t]{2}{*}{$\begin{array}{l}\text { Score of } \\
\text { arrhythmia }\end{array}$} \\
\hline & & & VF & VT & Other & Total & Brad. & \\
\hline Control & 8 & $2.9 \pm 0.7$ & $22.5 \pm 22.5$ & $18.9 \pm 9.1$ & $23.3 \pm 7.5$ & $64.7 \pm 29$ & $0 \pm 0$ & $2.1 \pm 0.6$ \\
\hline Control+ Gen & 7 & $1.6 \pm 0.8$ & $0 \pm 0$ & $13 \pm 6.7$ & $16.6 \pm 7.8$ & $34.3 \pm 16.6$ & $0 \pm 0$ & $1.3 \pm 0.4$ \\
\hline Ovx & 7 & $3.7 \pm 0.8$ & $6.04 \pm 6.04$ & $10.8 \pm 8.6$ & $17.8 \pm 7.8$ & $34.7 \pm 14.4$ & $0 \pm 0$ & $2.0 \pm 0.6$ \\
\hline Ovx + Gen & 7 & $3.1 \pm 0.7$ & $4.4 \pm 3.6$ & $27.3 \pm 12.4$ & $27.4 \pm 19.6$ & $59.1 \pm 27.5$ & $0 \pm 0$ & $2.3 \pm 0.6$ \\
\hline Sham & 5 & $3.9 \pm 1.1$ & $20 \pm 13.5$ & $24.2 \pm 9.8$ & $33.7 \pm 10.9$ & $78 \pm 10.3$ & $0 \pm 0$ & $3.4 \pm 0.8$ \\
\hline Sham + Gen & 5 & $1.8 \pm 0.7$ & $4.8 \pm 4.8$ & $11.8 \pm 6.4$ & $16.5 \pm 11.9$ & $33.1 \pm 16.7$ & $0 \pm 0$ & $2.0 \pm 0.8$ \\
\hline
\end{tabular}

Abbreviations: N, number of animals; VF, ventricular fibrillation; VT, ventricular tachycardia; Others; ventricular premature contraction, bigeminy, salvo; Brad., Bradycardia. Values were represented as mean \pm standard error

Table $\mathbf{5}$ The type and duration of arrhythmias during six minutes of reperfusion in acutely genistein administered group

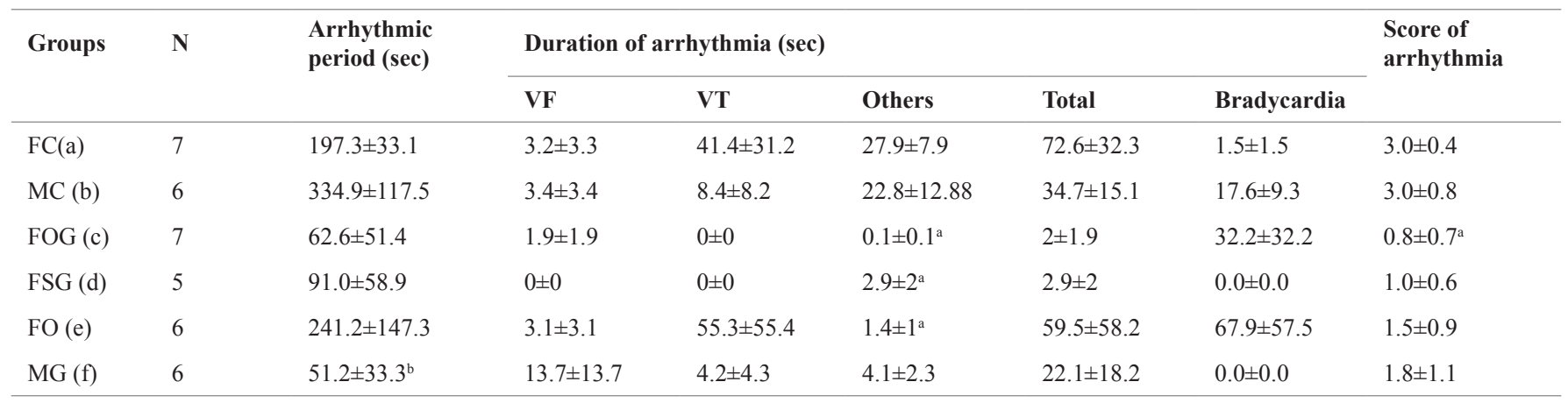

Abbreviations: FC, Female control; MC, Male control; FOG, Overiyectomised genistein administered female; FSG; Female sham group; FO, Overicetomised female; MG; Genistein administered male; N, number of animals. Values were represented as mean \pm standard error. abP $<0.05$; different from FC and MC.

\section{Discussion}

Major findings of this study are that genistein is not effective on the ischemia and reperfusion induced arrhythmia in acute and long-term administrations. These result support previous findings which also indicate genistein was not effective on ischemia induced arrhythmia., ${ }^{9,10}$ Another result of this study is that genistein did not significantly change the blood pressure and heart rate during reperfusion period in long-term administration. There is just one study in the literature that founds the effect of long-term genistein effect on the blood pressure and heart rate. ${ }^{2}$ In that study, two day genistein administration was not found to be effective on the blood pressure and heart rate. There are various studies indicating lower and higher doses of genistein have different effects on myocardial contractility, arrhythmia, and thyrosine kinase activity and infarct size. ${ }^{1-3,9,10}$ Contractility increases when the genistein was given in 30 and $50 \mathrm{ug} / \mathrm{kg}$ doses, decreases in $150 \mathrm{ug} / \mathrm{kg}$ dose. $^{2}$ In one of previous studies, it was indicated that $1 \mathrm{mg} / \mathrm{kg}$ dose of genistein given just before the reperfusion decreased ventricular fibrillation and premature contraction in ovariectomized female. ${ }^{1}$ However, in this study 45 minutes ischemia and 5hours reperfusion were made. This might be the reason of different result observed in our study. In other studies related with the effect of genistein on the ischemia/reperfusion-induced arrhythmia was made using langendorf perfusion method. ${ }^{9,10}$ In these studies, the genistein given alone was observed not to be effective on the total length of arrhythmia during 30 minutes of ischemia. It was observed that genistein abolishes the protective effect of ischemic preconditioning on the arrhythmias. ${ }^{9}$ The two studies above support the result of our study, although different method was used for coronary ligation. In our study, $1 \mathrm{mg} / \mathrm{kg}$ genistein did not affect the reperfusion-induced arrhythmia in ovariectomized rats in respect to sham operated animals. The decrease in arrhythmia in ovariectomized and sham operated genistein administered female in respect to control female is thought to occur due to the surgical operation made about seven days before coronary ligation. This preconditioning effect was not seen after four weeks following sham operation or ovariectomy. This result supports the finding that the injury produced in remote organ have preconditioning effect on the decreasing myocardial injury after the induced ischemic insult. ${ }^{14}$ Similarly, ischemia produced in remote organ decreases ischemia reperfusion arrhythmias. ${ }^{15}$ In the above two studies, the myocardial ischemia and reperfusion were produced after a short time following remote organ ischemia or injury. In our study, we did not aim to study the effect of remote organ injury on the ischemia or reperfusion induced arrhythmia. Nevertheless, obtained results indicate that the decreased arrhythmia in sham-operated female compared to the control female might depend on the preconditioning effect of remote organ injury. Low doses of genistein administration increases contractility in the heart muscle. ${ }^{2,16,17} \mathrm{We}$ expect that the blood pressure would increase due to the increased contractility of heart muscle, but in our study, acute gensitein administration just before reperfusion did not change the blood pressure. However, genistein increased basal blood pressure significantly in sham-genistein group and heart rate in the initial 
stage of reperfusion in long-term administration. This result indicates that long-term genistein administration increases the recovery of myocardium following reperfusion. ${ }^{16}$ Basal blood pressure and heart rate before coronary ligation are not changed in long-term genistein administered ovariectomized and unovariectomized females. This result did not support previous studies that genistein increases automatic depolarization in ventricular myocytes by inhibiting Kir current $^{8,18}$ and decreases spontaneous depolarization in SA node cells. ${ }^{19}$ The reason of differences with above study may be caused by smaller doses of genistein and different method used in our study.

\section{Conclusion}

This study obviously showed that genistein was not effective on the ischemia and reperfusion induced arrhythmias, blood pressure and heart rate both in acute or long-term administration in ovariectomized female and male rats.

\section{Acknowledgements}

This study was supported by the research project supported by Abant İzzet Baysal University Research Fund, Project numbers 2013.03.01.598; 2015.03.01.816.

\section{Conflict of interest}

The authors declare there is no conflict of interest.

\section{References}

1. Hayward CS, Kelly RP, Collins P. The roles of gender, the menopause and hormone replacement on cardiovascular function. Cardiovasc Res. 2000;46(1):28-49.

2. Mendelsohn ME, Karas RH. The protective effects of estrogen on the cardiovascular system. $N$ Engl J Med. 1999;340(23):801-1811.

3. Barrett-Connor E, Bush TL. Estrogen and coronary heart disease in women. JAMA. 1991;265(14):1861-1867.

4. Subbiah MT. Estrogen replacement therapy and cardioprotection: mechanisms and controversies. Braz J Med Biol Res. 2002;35(3):271-276.

5. Suetsugi M, Su L, Karlsberg $\mathrm{K}$, et al. Flavone and isoflavone phytoestrogens are agonists of estrogen-related receptors. Mol Cancer Res. 2003;1(13):981-991.

6. Carlson S, Peng N, Prasain JK, et al. Effects of botanical dietary supplements on cardiovascular, cognitive, and metabolic function in males and females. Gend Med. 2008;5 Suppl A:S76-S90.
7. Tissier R, Waintraub X, Couvreur N, et al. Pharmacological postconditioning with the phytoestrogen genistein. J Mol Cell Cardiol. 2007;42(1):79-87.

8. Lissin LW, Cooke JP. Phytoestrogens and cardiovascular health. J Am Coll Cardiol. 2000;35(6):1403-1410.

9. Fatehi-Hassanabad Z, Parratt JR. Genistein, an inhibitor of tyrosine kinase, prevents the antiarrhythmic effects of preconditioning. Eur J Pharmacol. 1997;338(1):67-70.

10. Söylemez S, Demiryürek AT, Kanzik I. Involvement of tyrosine kinase in peroxynitrite-induced preconditioning in rat isolated heart. Eur $J$ Pharmacol. 2003;464(2-3):163-169.

11. Bozdogan O, Kaya ST, Yasar S, et al. Short-term diabetes decreases ischemia reperfusion-induced arrhythmia: the effect of alpha-2 blocker yohimbine and glibenclamide. Turkish Journal of Biology. 2016;40(4):899-905.

12. Curtis MJ, Hancox JC, Farkas A, et al. The Lambeth Conventions (II): guidelines for the study of animal and human ventricular and supraventricular arrhythmias. Pharmacol Ther. 2013;139(2):213-248.

13. Leprán I, Baczkó I, Varró A, et al. ATP-sensitive potassium channel modulators: both pinacidil and glibenclamide produce antiarrhythmic activity during acute myocardial infarction in conscious rats. J Pharmacol Exp Ther. 1996;277(3):1215-1220.

14. Song Y, Ye YJ, Li PW, et al. The cardioprotective effects of late-phase remote preconditioning of trauma depends on neurogenic pathways and the activation of PKC and NF-kappaB (But Not iNOS) in mice. J Cardiovasc Pharmacol Ther. 2016;21(3):310-319.

15. Noorbakhsh MF, Arab HA, Kazerani HR. Liver ischemia preconditions the heart against ischemia-reperfusion arrhythmias. Iran J Basic Med Sci. 2015;18(1):80-88.

16. Deodato B, Altavilla D, Squadrito G, et al. Cardioprotection by the phytoestrogen genistein in experimental myocardial ischaemia-reperfusion injury. Br J Pharmacol. 1999;128(8):1683-1690.

17. Li H, Zhang Y, Tian Z, et al. Genistein stimulates myocardial contractility in guinea pigs by different subcellular mechanisms. Eur J Pharmacol. 2008;597(1-3):70-74.

18. Chiang CE, Luk HN, Chen LL, et al. Genistein inhibits the inward rectifying potassium current in guinea pig ventricular myocytes. $J$ Biomed Sci. 2002;9(4):321-326.

19. Ma T, Fan ZZ, He RR. Electrophysiological effects of phytoestrogen genistein on pacemaker cells in sinoatrial nodes of rabbits. Acta Pharmacol Sin. 2002;23(4):367-370 